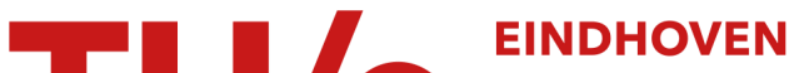 \\ UNIVERSITY OF \\ TECHNOLOGY
}

\section{Decoupling of bidiagonal systems involving singular blocks}

\section{Citation for published version (APA):}

Mattheij, R. M. M. (1989). Decoupling of bidiagonal systems involving singular blocks. (RANA : reports on applied and numerical analysis; Vol. 8925). Technische Universiteit Eindhoven.

\section{Document status and date:}

Published: 01/01/1989

\section{Document Version:}

Publisher's PDF, also known as Version of Record (includes final page, issue and volume numbers)

\section{Please check the document version of this publication:}

- A submitted manuscript is the version of the article upon submission and before peer-review. There can be important differences between the submitted version and the official published version of record. People interested in the research are advised to contact the author for the final version of the publication, or visit the $\mathrm{DOI}$ to the publisher's website.

- The final author version and the galley proof are versions of the publication after peer review.

- The final published version features the final layout of the paper including the volume, issue and page numbers.

Link to publication

\section{General rights}

Copyright and moral rights for the publications made accessible in the public portal are retained by the authors and/or other copyright owners and it is a condition of accessing publications that users recognise and abide by the legal requirements associated with these rights.

- Users may download and print one copy of any publication from the public portal for the purpose of private study or research.

- You may not further distribute the material or use it for any profit-making activity or commercial gain

- You may freely distribute the URL identifying the publication in the public portal.

If the publication is distributed under the terms of Article 25fa of the Dutch Copyright Act, indicated by the "Taverne" license above, please follow below link for the End User Agreement:

www.tue.nl/taverne

Take down policy

If you believe that this document breaches copyright please contact us at:

openaccess@tue.nl

providing details and we will investigate your claim. 
RANA 89-25

December 1989

DECOUPLING OF BIDIAGONAL

SYSTEMS INVOLVING

SINGULAR BLOCKS

by

R.M.M. Mattheij

Reports on Applied and Numerical Analysis

Department of Mathematics and Computing Science

Eindhoven University of Technology

P.O. Box 513

5600 MB Eindhoven

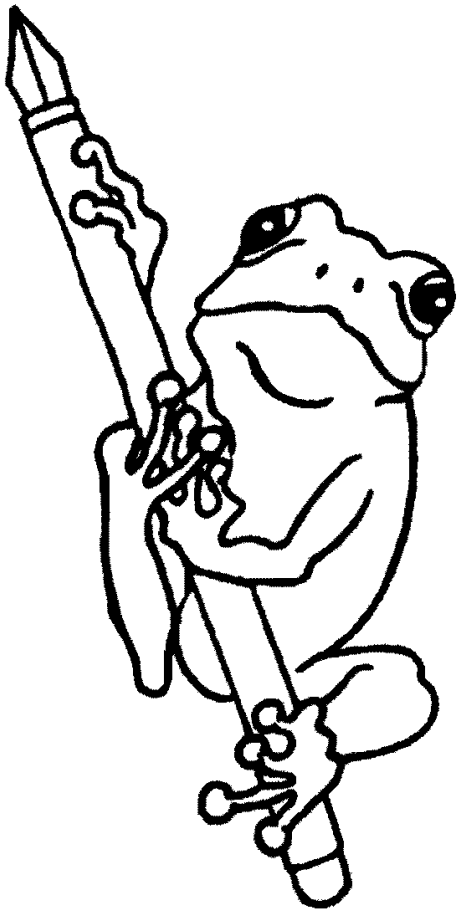

The Netherlands 


\title{
DECOUPLING OF BIDIAGONAL SYSTEMS \\ INVOLVING SINGULAR BLOCKS
}

\author{
R.M.M. Mattheij \\ Faculteit Wiskunde en Informatica \\ Technische Universiteit Eindhoven \\ Postbus 513 \\ 5600 MB Eindhoven \\ The Netherlands
}

\begin{abstract}
For one step difference equations, where the matrix coefficients may be singular, a stability analysis based on using fundamental solutions and their inverses does not apply. This paper shows how well-boundedness of the Green's function leads to a kind of dichotomy of the fundamental solution, including certain "parasitic solutions" (which arise because of the singularity of the fundamental solutions). This then is used to show how one can find a stable decoupling and thus a numerical algorithm for solving a discrete BVP. Several examples sustain the analysis.
\end{abstract}




\section{Introduction}

In analyzing numerical methods for boundary value problems, one often uses explicit representations or estimates of Green's functions. The notion of well-conditioning of such a BVP is intimately connected to a moderate bound of these Green's functions. As a consequence one can show cf. [6] that there exists a natural splitting of the solution space into nongrowing and nondecaying solutions. It is essential for the latter analysis that the fundamental solution is nonsingular everywhere. A discrete analogue of such an analysis is straight-forward (cf. [2]).

However, if the differential equations are implicit, and have an index $\geq 1$, i.e. if in the (linearized) system, the coefficient matrix of the derivative term is allowed to be singular, this may lead to problems. These differential algebraic equations are in fact ODEs coupled with algebraic relations, cf. [5]. For certain numerical methods the rank deficiency is inherited in some of the matrix coefficients of the difference equation. This then explains our interest in rank deficient coefficients. Another example of one step recursions with such a rank deficiency arises from discretization of stiff problems; e.g. if we have

$$
\frac{d x}{d t}=\lambda(t) x, \lambda \in \mathbb{R}
$$

and we use an implicit method, the trapezezoidal rule say, then the resulting recursion reads

$$
\left(1-h_{i} \lambda\left(t_{i+1}\right)\right) x_{i+1}=\left(1+h_{i} \lambda\left(t_{i}\right)\right) x_{i}
$$

Clearly for $h_{i} \lambda\left(t_{i+1}\right)=1$ or $h_{i} \lambda\left(t_{i}\right)=-1$ either one of the coefficients is zero. A generalisation to systems is obvious.

In the singular coefficient case, we no longer have a fundamental solution that is invertible everywhere. This then makes the more or less standard argumentation for relating well-conditioning to dichotomy no longer applicable. Indeed, apparently some "parasitic" solutions are generated (filling in for the trivial solutions in the Green's function); we have to show that these behave well in some sense too.

In this paper we shall exclusively deal with the discrete rank deficient case. We shall analyze the Green's function and draw conclusions with respect to growth of ("parasitic") solutions. As a major result we can thus show how and why a decoupling technique makes sense and is stable for such problems. The analysis is in particular applied to problems which involve block tridiagonal matrices as arise e.g. from collocation methods for BVP. However, they may also be useful for multiple shooting techniques and related methods.

In section 2 we generalise concepts and estimates for Green's functions for the singular case. In section 3 we then show what can be said about the growth of basis modes (as far as they exist). As a special application of this analysis we consider in section 4 tridiagonal matrices arising from a particular repartitioning in a staircase matrix, thus inducing singular blocks. The analysis finally enables us to derive a stable decoupling algorithm in section 5 . The theory is illustrated by some 
$-3-$

examples in section 6. 


\section{Green's functions and their consequences}

Let us consider a general implicit one step recursion for $\left\{x_{i}\right\}_{i=1}^{N}$

$$
A_{i} x_{i}+B_{i} x_{i+1}=f_{i}, i=1, \ldots, N-1,
$$

where $A_{i}, B_{i} \in \mathbb{R}^{n \times n}, f_{i} \in \mathbb{R}^{n}$. Assume we have a boundary condition $(B C)$ for $\left\{x_{i}\right\}$ given by

(2.1b) $\quad M_{1} x_{1}+M_{N} x_{N}=b$,

$M_{1}, M_{N} \in \mathbb{R}^{n \times n}, b \in \mathbb{R}^{n}$.

The problem (2.1) can be written as an $(n \times N)^{2}$ matrix equation

(2.2a) $\quad \mathbf{A x}=\mathbf{b}$,

where

$$
\mathbf{A}:=\left[\begin{array}{ccccc}
A_{1} & B_{1} & & & \\
& A_{2} & B_{2} & & \\
& & \ddots & \ddots & \\
& & & A_{N-1} & B_{N-1} \\
M_{1} & & & & M_{N}
\end{array}\right], \mathbf{x}:=\left[\begin{array}{c}
x_{1} \\
\vdots \\
\vdots \\
\vdots \\
x_{N}
\end{array}\right), \mathbf{b}:=\left[\begin{array}{c}
f_{1} \\
\vdots \\
\vdots \\
f_{N-1} \\
\mathbf{b}
\end{array}\right] .
$$

The conditioning (with respect to absolute errors) is then characterized by the conditioning constant $\mathrm{x}$, defined as (cf. [2])

$$
\left\|\mathbf{A}^{-1}\right\|=: \kappa
$$

We assume (2.1) to be well posed, i.e. $\mathbf{A}$ is invertible. Then there exists a fundamental solution $\left\{\Phi_{i}\right\}_{i=0}^{N}$ with

$$
\begin{aligned}
& A_{i} \Phi_{i}+B_{i} \Phi_{i+1}=0 \\
& M_{1} \Phi_{1}+M_{N} \Phi_{N}=I .
\end{aligned}
$$

Also, there exists a Green's function $\left\{G_{i j}\right\}_{i=1}^{N}, j=1, \ldots, N-1$ with

$$
A_{i} G_{i j}+B_{i} G_{i+1, j}=\delta_{i j} I
$$

$$
M_{1} G_{i j}+M_{N} G_{N j}=0
$$

Explicit expressions for $\left\{G_{i j}\right\}$ when $A_{i} \equiv I$ and $B_{i}$ is nonsingular for all $i$ can be found e.g. in [7]. In this section we shall derive, more generally, expressions for the potentially rank deficient case, i.e. we assume that for at least one index $j$ a matrix $A_{j}$ or $B_{j}$ is singular, i.e.

$$
A_{j} \Phi_{j}=-B_{j} \Phi_{j+1}
$$

is singular.

If we denote by $A^{+}$the pseudo-inverse of a matrix $A$ (cf. [4]), then 


$$
B_{j} \Phi_{j+1}\left(B_{j} \Phi_{j+1}\right)^{+}=: P_{j}
$$

defines an orthogonal projection $\mathbb{R}^{n} \rightarrow \operatorname{Range}\left(B_{j} \Phi_{j+1}\right)$. In order to construct the Green's function we make the Ansatz

$$
G_{i j}=\Phi_{i} K_{j}+F_{i j}, i \leq j \quad K_{j} \in \mathbb{R}^{n^{2}}
$$

$$
G_{i j}=\Phi_{i} L_{j}+F_{i j}, i \leq j \quad L_{j} \in \mathbb{R}^{n^{2}}
$$

where $\left(1-P_{j}\right) F_{i j}=0$. (Note that $F_{i j}=0$ is the nonsingular case.) From (2.4a) and (2.7) we see

$$
A_{j} \Phi_{j} K_{j}+B_{j} \Phi_{j+1} L_{j}=P_{j}
$$

$$
A_{j} F_{j j}+B_{j} F_{j, j+1}=I-P_{j}
$$

Using (2.5) we obtain

$$
B_{j} \Phi_{j+1}\left(L_{j}-K_{j}\right)=P_{j}
$$

and take

$$
\left(L_{j}-K_{j}\right)=\left(B_{j} \Phi_{j+1}\right)^{+} .
$$

Now we find from (2.4b) (N.B. $M_{1} \Phi_{1}=1-M_{N} \Phi_{N}$ )

$$
M_{N} \Phi_{N}\left(L_{j}-K_{j}\right)=-K_{j},
$$

whence

$$
K_{j}=-M_{N} \Phi_{N}\left(B_{j} \Phi_{j+1}\right)^{+} .
$$

A similar derivation can be given for $L_{j}$. Summarizing we have

Property 2.13. Define $\bar{G}_{i j}=G_{i j} P_{j}$, then

$$
\bar{G}_{i j}=\Phi_{i} M_{N} \Phi_{N}\left(A_{j} \Phi_{j}\right)^{+}, i \leq j
$$

$$
\tilde{G}_{i j}=\Phi_{i} M_{1} \Phi_{1}\left(B_{j} \Phi_{j+1}\right)^{+}, i>j \text {. }
$$

\section{Property 2.14.}

(i)

$$
\begin{aligned}
& \left\|\Phi_{i} M_{N} \Phi_{N} \Phi_{j}^{+}\right\| \leq \kappa\left\|A_{j}\right\|, \quad i \leq j \\
& \left\|\Phi_{i} M_{1} \Phi_{1} \Phi_{j+1}^{+}\right\| \leq \kappa\left\|B_{j}\right\|, \quad i \geq j+1
\end{aligned}
$$

Proof. First we show that if $A_{j} \Phi_{j} y=0$ for some nontrivial vector $y$, then $\Phi_{j+1} y=0$. Suppose the latter is not true; without restriction assume $\|y\|_{2}=1$. Let $Y$ be an orthogonal matrix with $y$ as its first column and $Y(\alpha)$ equal to $Y$ but for its first column which is $\alpha y, \alpha \in \mathbb{R}$. Define another matrix solution $\left\{\Psi_{i}(\alpha)\right\}$, where $\Psi_{i}(\alpha)=\Phi_{i} Y, i \leq j$ and $\Psi_{i}(\alpha)=\Phi_{i} Y(\alpha), i \geq j+1$. (Note that in particular $A_{j} \Psi_{j}(\alpha)=-B_{j} \Psi_{j+1}(\alpha)$.) We have 


$$
\begin{aligned}
M_{1} \Psi_{1}(\alpha)+M_{N} \Psi_{N}(\alpha) & =M_{1} \Phi_{1} Y+M_{N} \Phi_{N} Y(\alpha) \\
& =Y+(\alpha-1) M_{N} \Phi_{N}\left[\begin{array}{lllll}
y & 0 & \cdots & 0
\end{array}\right]
\end{aligned}
$$

Hence there clearly exists a choice for $\alpha \neq 1$ such that $M_{1} \Psi_{1}(\alpha)+M_{N} \Psi_{N}(\alpha)$ is nonsingular, showing that $\left\{\Psi_{i}(\alpha)\right\}$ is another fundamental solution. However, since $\Psi_{1}(\alpha) Y^{-1}=\Phi_{1}$ we can't have uniqueness unless $\Phi_{i} y=0, i=j+1, \ldots, N$.

So for the rest of the proof we can therefore restrict ourselves to Range $\left(A_{j} \Phi_{j}\left(A_{j} \Phi_{j}\right)^{+}\right)=\operatorname{Range}\left(B_{j} \Phi_{j+1}\left(B_{j} \Phi_{j+1}\right)^{+}\right)=: S_{j}$, i.e. for (ii) we deduce from the foregoing that if $u \in \operatorname{range}\left(\Phi_{j+1} \Phi_{j+1}^{+}\right) \Rightarrow B_{j} \Phi_{j+1} u \neq 0$. We can then proceed as follows

$$
\left\|\Phi_{i} M_{1} \Phi_{1}\left(B_{j} \Phi_{j+1}\right)^{+}\right\|=\max _{z \neq 0} \frac{\left\|\Phi_{i} M_{1} \Phi_{1}\left(B_{j} \Phi_{j+1}\right)^{+} z\right\|}{\|z\|}
$$

It is not restrictive to assume $B_{j} \Phi_{j+1}\left(B_{j} \Phi_{j+1}\right)^{+} z=z$, so with $\left(B_{j} \Phi_{j+1}\right)^{+} z=: y$, i.e. $B_{j} \Phi_{j+1} y=z$, we find

$$
\left\|\Phi_{i} M_{1} \Phi_{1}\left(B_{j} \Phi_{j+1}\right)^{+}\right\|=\max _{y \neq 0} \frac{\left\|\Phi_{i} M_{1} \Phi_{1} y\right\|}{\left\|B_{j} \Phi_{j+1} y\right\|} \geq \max _{y \neq 0} \frac{\left\|\Phi_{i} M_{1} \Phi_{1} y\right\|}{\left\|B_{j}\right\|\left\|\Phi_{j+1} y\right\|} .
$$

Since $\Phi_{j+1} y=: u \neq 0$, we thus see that the last expression is underestimated by

$$
\max _{u \neq 0} \frac{\left\|\Phi_{i} M_{1} \Phi_{1} \Phi_{j+1}^{+} u\right\|}{\left\|B_{j}\right\|\|u\|}=\frac{\left\|\Phi_{i} M_{1} \Phi_{1} \Phi_{j+1}^{+}\right\|}{\left\|B_{j}\right\|},
$$

from which (ii) follows. Estimate (i) goes similarly.

Clearly, if all $A_{j}$ and $B_{j}$ are nonsingular, the expressions in (2.13) coincide with the standard form Green's function.

Next, we consider the complementary parts (see (2.7) and (2.8b)).

\section{Property 2.15 .}

(i) $\left\{F_{i j}\right\}_{i \leq j}$ and $\left\{F_{i j}\right\}_{j z i+1}$ satisfy the homogeneous recursion of (2.1).

(ii) $\operatorname{Range}\left(B_{j} F_{j, j+1}\right) \cap \operatorname{Range}\left(A_{j} F_{j j}\right)=\{0\}$.

(iii) There exists an orthogonal projection $Q_{j}$ such that

$$
\begin{aligned}
& B_{j} F_{j, j+1}=\left(I-P_{j}\right) Q_{j} \\
& A_{j} F_{j j}=\left(I-P_{j}\right)\left(I-Q_{j}\right) .
\end{aligned}
$$

Proof. (ii): If $\operatorname{Range}\left(B_{j} F_{j, j+1}\right) \cap \operatorname{Range}\left(A_{j} F_{j j}\right) \neq\{0\}$, then there exist vectors $a$ and $b$ such that $A_{j} F_{j j} a+B_{j} F_{j, j+1} b=0$ with $A_{j} F_{j j} a \neq 0$. Now define $\left[f_{i}\right\}$ by 


$$
\begin{aligned}
f_{i}=F_{i j} a, & i \leq j \\
f_{i}=F_{i j} b, & i \geq j+1 .
\end{aligned}
$$

Then clearly $\left\{f_{i}\right\}$ satisfies the homogeneous recursion for $i=j$ and on account of (i) also for $i \neq j$. So there exists a vector $c$ such that $f_{i}=\Phi_{i} c$ for all $i$, and in particular for $i=j$. Hence $A_{j} \Phi_{j} c=A_{j} F_{j j} a \neq 0$. Since $A_{j} \Phi_{j} c \in \operatorname{Range}\left(P_{j}\right)$ and $\left(A_{j} F_{j j}\right) \in \operatorname{Range}\left(1-P_{j}\right)$ this is clearly a contradiction

(iii) Define $Q_{j}$ by $\left(I-P_{j}\right) Q_{j}=B_{j} F_{j, j+1}\left(B_{j} F_{j, j+1}\right)^{+}$(cf. (2.6)).

Property 2.16. The splitting for $G_{i j}$ in (2.7) is unique and hence $\tilde{G}_{i j}$ is uniquely defined by (2.13a,b).

Proof. Clearly $\bar{G}_{i j}$ is uniquely defined. Now suppose $\left\{H_{i j}\right\}$ is defined as $\left\{F_{i j}\right\}$ in (2.7). Then $\left\{F_{i j}-H_{i j}\right\}$ satisfies the homogeneous recursion. Hence for some matrix $S$ we have

$$
F_{i j}-H_{i j}=\Phi_{i} S
$$

Since we must have $M_{1} F_{1 j}+M_{N} F_{N j}=M_{1} H_{1 j}+M_{N} H_{N j}=0$ (see (2.4b)) we deduce $\left(M_{1} \Phi_{1}+M_{N} \Phi_{N}\right) S=0 \Rightarrow S=0$, so $F_{i j}=H_{i j}$.

We find immediately:

Corollary 2.17. $\left\|\tilde{G}_{i j}\right\|_{2},\left\|F_{i j}\right\|_{2} \leq\left\|G_{i j}\right\|_{2}$. 


\section{Estimates for the fundamental solutions}

In section 2 we derived expressions for the Green's function in terms of the fundamental solution $\left\{\Phi_{i}\right\}$ and certain parasitic solutions $\left\{F_{i j}\right\}_{j \text { fixed }}$. The latter kind of "solutions" are effectively onesided solutions, i.e. built up from components which satisfy the homogeneous recursion for either $i>j$ or $i \leq j$; this is in contrast to the fundamental solution which exists globally (albe it with possible zero components on a range of consecutive indices).

We shall now show that well conditioning of the BVP (2.1), (2.2) implies a kind of dichotomy of $\left\{\Phi_{i}\right\}$ and a kind of one sided stability for $\left\{F_{i j}\right\}$. To do this we first have to generalise some results of [6]; not so much because our problem is discrete, but rather because it is singular.

First we examine the structure of $\left\{\Phi_{i}\right\}$ more in detail. Let $\operatorname{rank}\left(\Phi_{1}\right)=n-l$ and $\operatorname{rank}\left(\Phi_{N}\right)=n-m$. (N.B. $0<l+m \leq n$.) Then there clearly exists an orthogonal $V$ such that

$$
\left.\Phi_{1} V=[*] \underset{i}{\varnothing}\right]
$$

i.e. the last $l$ columns of $\Phi_{1} V$ consist of zeros; consequently the last columns of $\Phi_{N} V$ must have full rank. However if $m>0$, we may postmultiply $V$ by an orthogonal matrix $W$,

$$
W=\left[\begin{array}{cc}
\hat{W} & 0 \\
0 & I_{l}
\end{array}\right], \hat{W} \in \mathbb{R}^{(n-l)^{2}},
$$

such that

$$
\left.\Phi_{N} V W=\underset{m}{[\varnothing \mid} *\right]
$$

It is therefore not restrictive to identify $V$ and $V W$. Next, let $U_{1}$ be an orthogonal matrix such that

$$
U_{1}^{T} \Phi_{1} V=\left[\begin{array}{lrr} 
& \square & \varnothing \\
\varnothing & \varnothing \\
\varnothing & \varnothing & \varnothing
\end{array}\right] \unlhd_{l}=: \Psi_{1}
$$

(i.e. $\Psi_{1}$ is upper triangular) and $U_{N}$ an orthogonal matrix such that

$$
U_{N}^{T} \Phi_{N} V=\left[\begin{array}{lll}
\varnothing & \varnothing & \varnothing \\
\varnothing & \varnothing \\
\varnothing & \square &
\end{array}\right] \downarrow_{l}=: \Psi_{N}
$$

(i.e. $\Psi_{N}$ is lower triangular). Let $\Phi_{1}$ be a special initial value defined by (cf. (3.2a))

$$
\Phi_{1}:=\left\{\Psi_{1}+\left[\begin{array}{lll}
\varnothing & \varnothing & \varnothing \\
\varnothing & \varnothing & \varnothing \\
\varnothing & \varnothing & I
\end{array}\right]\right\} V^{T}
$$

It is simple to verify that 


$$
\Phi_{N} \bar{\Phi}_{1}^{-1}=U_{N}^{T}\left[\begin{array}{lll}
\varnothing & \varnothing & \varnothing \\
\varnothing & A & \varnothing \\
\varnothing & B & C
\end{array}\right],
$$

for some $A \in \mathbb{R}^{(n-m-l)^{2}}, B \in \mathbb{R}^{(n-m) \times l}$ and $C \in \mathbb{R}^{l \times l}, A$ and $C$ being nonsingular. As a following step let

(3.4a) $\quad A=: \bar{U} \bar{\Sigma} \bar{v}^{T}$,

be the SVD of $A$, where $\tilde{\Sigma}$ has positive elements $\sigma_{1}, \ldots, \sigma_{n-m-l}$. Assume $0<\sigma_{1}, \ldots, \sigma_{k} \leq 1$ and $\sigma_{k+1}, \ldots, \sigma_{n-m-l}>1$, for some $k \in \mathbb{N}$. Like in [6] we split $\bar{\Sigma}$ in two parts (which effectively corresponds to a separation of non-increasing and increasing modes coupled by the boundary condition); so we introduce

(3.4c) $\quad \tilde{D}=\left[\begin{array}{cc}I_{k} & \varnothing \\ \varnothing & D_{2}\end{array}\right]$.

Inspired by this partitioning, let a projection $P$ be given by

$$
P:=\left[\begin{array}{cc}
I_{m+k} & \varnothing \\
\varnothing & \varnothing
\end{array}\right] \text {. }
$$

Finally, let

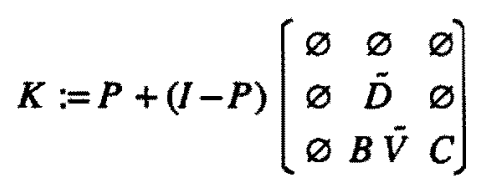

(note that $K$ is nonsingular).

Then we define a fundamental solution $\left\{\tilde{\Phi}_{i}\right\}$ by

$$
\tilde{\Phi}_{i}:=\Phi_{i} \bar{\Phi}_{1}^{-1} X K^{-1}
$$

where

$$
X:=\left[\begin{array}{lll}
I_{m} & \varnothing & \varnothing \\
\varnothing & \tilde{V} & \varnothing \\
\varnothing & \varnothing & I_{l}
\end{array}\right],
$$

and separated $\mathrm{BC} \bar{M}_{1}, \bar{M}_{N}$ by

$$
\tilde{M}_{1}:=P X^{T} U_{1}^{T}
$$

$$
\tilde{M}_{N}:=(I-P) Y^{T} U_{N}^{T}
$$

where 
(3.8c) $\quad Y:=\left[\begin{array}{ccc}I_{m} & \varnothing & \varnothing \\ \varnothing & \tilde{U} & \varnothing \\ \varnothing & \varnothing & I_{l}\end{array}\right]$.

We have the following lemmata

\section{Lemma 3.9.}

(i)

$$
\bar{M}_{1} \bar{\Phi}_{1}+\bar{M}_{N} \bar{\Phi}_{N}=I
$$

(ii) $\quad\left\|\bar{M}_{1}\right\|_{2} \leq 1,\left\|\bar{M}_{N}\right\|_{2} \leq 1$

(iii) $\quad\left\|\tilde{\Phi}_{1}\right\|_{2} \leq 1,\left\|\bar{\Phi}_{N}\right\|_{2} \leq 1$

Proof. We have

$$
\tilde{\Phi}_{1}=\Phi_{1} \bar{\Phi}_{1}^{-1} X K^{-1}=U_{1}\left[\begin{array}{ccc}
I_{m} & \varnothing & \varnothing \\
\varnothing & \bar{V} & \varnothing \\
\varnothing & \varnothing & \varnothing
\end{array}\right]\left[\begin{array}{ccc}
I_{m} & \varnothing & \varnothing \\
\varnothing & \bar{D}^{-1} & \varnothing \\
\varnothing & \bar{E} & C^{-1}
\end{array}\right],
$$

where $\bar{E}=-C^{-1} B \bar{V} \bar{D}^{-1}$.

Because of the construction of $\bar{D}^{-1}$ it directly follows that $\left\|\bar{\Phi}_{1}\right\|_{2} \leq 1$. Furthermore we have

$$
\tilde{\Phi}_{N}=\Phi_{N} \bar{\Phi}_{1}^{-1} X K^{-1}=U_{N}^{T} Y\left[\begin{array}{lcc}
\varnothing & \varnothing & \varnothing \\
\varnothing & \bar{\Sigma} \tilde{D}^{-1} & \varnothing \\
\varnothing & \varnothing & l_{l}
\end{array}\right] \text {. }
$$

Hence $\left\|\tilde{\Phi}_{N}\right\|_{2} \leq 1$. From these expressions of $\tilde{\Phi}_{1}$ and $\tilde{\Phi}_{N}$ the other assertions follow immediately.

Lemma 3.10. $\left\|\tilde{\Phi}_{i}\right\| \leq 2 \kappa$.

Proof.

$$
\tilde{\Phi}_{i}=\Phi_{1}\left[M_{1} \Phi_{1}+M_{N} \Phi_{N}\right]\left[\bar{\Phi}_{1}^{-1} X K^{-1}\right]=\Phi_{i} M_{1} \tilde{\Phi}_{1}+\Phi_{i} M_{N} \tilde{\Phi}_{N}
$$

So the estimate follows from Lemma 3.9.

Theorem 3.11. $\left\|\tilde{\Phi}_{i} P \bar{\Phi}_{j}^{+}\right\| \leq\left(\kappa+4 \kappa^{2}\right) \gamma_{j}, i \leq j$,

$$
\begin{aligned}
& \left\|\bar{\Phi}_{i}(1-P) \bar{\Phi}_{j+1}^{+}\right\| \leq\left(\kappa+4 \kappa^{2}\right) \gamma_{j}, i \geq j+1, \\
& \text { where } \gamma_{j}=\max \left(\left\|A_{j}\right\|,\left\|B_{j}\right\|\right) .
\end{aligned}
$$

Proof. We only show the result for $i \geq j+1$. Let $S^{-1}:=\bar{\Phi}_{1}^{-1} X K^{-1}$ (cf. (3.7a)) then $\tilde{\Phi}_{j+1}^{+}=S M_{1} \Phi_{1} \Phi_{j+1}^{+}+S M_{N} \Phi_{N} \Phi_{j+1}^{+}$. So 


$$
\begin{aligned}
\ddot{\Phi}_{i} \bar{M}_{1} \tilde{\Phi}_{1} \tilde{\Phi}_{j+1}^{+} & =\tilde{\Phi}_{i}\left(1-\tilde{M}_{N} \tilde{\Phi}_{N}\right) S M_{1} \Phi_{1} \Phi_{j+1}^{+}+\tilde{\Phi}_{i}\left(\tilde{M}_{1} \tilde{\Phi}_{1}\right) S M_{N} \Phi_{N} \bar{\Phi}_{j+1}^{+} \\
& =\left[\Phi_{i} M_{1} \Phi_{1} \Phi_{j+1}^{+}\right]-\tilde{\Phi}_{i} \tilde{M}_{N}\left[\Phi_{N} M_{1} \Phi_{1} \Phi_{j+1}^{+}\right]+\tilde{\Phi}_{i} \tilde{M}_{1}\left[\Phi_{1} M_{N} \Phi_{N} \Phi_{j+1}^{+}\right]
\end{aligned}
$$

The expressions in square brackets are each bounded in norm by $\kappa \gamma_{j}$. Since $\tilde{M}_{1} \tilde{\Phi}_{1}=P$, the result follows from using Lemma 3.9, Lemma 3.10 and Theorem 2.15.

We conclude from this theorem that there exists a "dichotomic" fundamental solution $\left\{\tilde{\Phi}_{i}\right\}$ in the sense that basis modes of

(3.12a) $\quad \mathbf{S}_{1}:=\left\{\tilde{\Phi}_{i} P c \mid c \in \mathbb{R}\right\}$

do not increase significantly, as long as they exist for increasing index, and similarly of

$$
\mathbf{S}_{2}:=\left\{\tilde{\Phi}_{i}(I-P) c \mid c \in \mathbb{R}\right\},
$$

for decreasing index.

As far as parasitic modes (i.e. which do not exist till some point) is concemed, we deduce from Property 2.17

Theorem 3.13. For any index $j$ for which $P_{j} \neq I$ (see (2.6)) we have

$$
\begin{aligned}
& \left\|F_{i j}\right\|_{2} \leq \kappa, \quad i>j \\
& \left\|F_{i j}\right\|_{2} \leq \kappa, \quad i \leq j .
\end{aligned}
$$

So these parasitic modes do not grow, away from the point $j$ from which they originate.

Remark 3.14. If the BC are separated we do not have to construct the fundamental solution $\bar{\Phi}$ as above. As in [6] the projection matrix $P$ can then be identified with $M_{1} \Phi_{1}$. 


\section{Application to special block tridiagonal matrices}

Consider a discrete two point BVP with separated BC, i.e. in (2.1b) we have

$$
M_{1}=\left[\begin{array}{c}
M_{1}^{2} \\
\varnothing
\end{array}\right]^{I_{k}}, M_{N}=\left(\begin{array}{c}
\varnothing \\
M_{N}^{1}
\end{array}\right] I_{n-k}
$$

A natural way of employing this zero structure (which apparently induces a decoupling) is to write the resulting system for $x_{1}, \ldots, x_{N}$ as

(4.2a) $\quad \mathbf{A x}=\mathbf{b}$,

where

(4.2b)

$$
\begin{aligned}
& \mathbf{A}=\left[\begin{array}{ccccc}
M_{1}^{2} & & & & \\
A_{1} & B_{1} & & & \varnothing \\
& A_{2} & B_{2} & & \\
& & \ddots & \ddots & \\
& \varnothing & & A_{N-1} & B_{N-1} \\
& & & & M_{N}^{1}
\end{array}\right], \\
& \mathbf{x}=\left[\begin{array}{c}
x_{1} \\
\cdot \\
\cdot \\
x_{N}
\end{array}\right], \quad \mathbf{b}=\left[\begin{array}{c}
b^{2} \\
f_{1} \\
\vdots \\
f_{N-1} \\
f_{N-1} \\
b^{1}
\end{array}\right]=\left[\begin{array}{c}
b_{1} \\
\cdot \\
\cdot \\
\cdot \\
b_{N}
\end{array}\right]
\end{aligned}
$$

(here $b^{2}, b^{1}$ denote the first $k$ and last $(n-k)$ coordinates of $b$ ). We can repartition the matrix $\mathbf{A}$ into a block tridiagonal one (with square blocks of size $n$ ). This results in a two step recursion

$$
S_{i} x_{i-1}+Q_{i} x_{i}+R_{i} x_{i+1}=b_{i}, i=2, \ldots, N-1
$$

where (with obvious row partioning for $A_{i}, B_{i}, C_{i}$ )

$$
S_{i}=\left[\begin{array}{c}
A_{i-1}^{2} \\
\varnothing
\end{array}{ }^{I_{k}}, Q_{i}=\left[\begin{array}{c}
B_{i-1}^{2} \\
A_{i}^{1}
\end{array}\right]{ }^{I_{k}}, R_{i}=\left[\begin{array}{c}
\varnothing \\
B_{i}^{1}
\end{array}\right] \unlhd_{k} .\right.
$$

Moreover we obtain a (separated) BC

$$
\left[\begin{array}{c}
M_{1}^{2} \\
A_{1}^{1}
\end{array}\right] x_{1}+\left[\begin{array}{c}
\varnothing \\
B_{1}^{1}
\end{array}\right] x_{2}=\left[\begin{array}{c}
b^{2} \\
f^{1}
\end{array}\right]{ }_{k}
$$




$$
\left[\begin{array}{c}
A_{N-1}^{2} \\
\varnothing
\end{array}\right] x_{N-1}+\left[\begin{array}{c}
B_{N-1}^{2} \\
M_{N}^{1}
\end{array}\right] x_{N}=\left[\begin{array}{c}
f_{N-1}^{2} \\
b^{1}
\end{array}\right] \downarrow_{k}
$$

The recursion (4.3) was already discussed in [8] with respect to stability and dichotomy by rewriting it into one step form:

$$
\left[\begin{array}{cc}
I_{n} & \varnothing \\
\varnothing & R_{i}
\end{array}\right]\left[\begin{array}{c}
x_{i} \\
x_{i+1}
\end{array}\right]+\left[\begin{array}{cc}
\varnothing & -I_{n} \\
S_{i} & Q_{i}
\end{array}\right]\left[\begin{array}{c}
x_{i-1} \\
x_{i}
\end{array}\right]+\left[\begin{array}{c}
0 \\
b_{i}
\end{array}\right] .
$$

Clearly both matrices in this implicit recursion are singular; this caused some problems for application of the general theory, which were circumvented by an indirect analysis in [8].

With the more general results of the preceding sections we can tackle this problem more naturally now. Rather than (4.6) we consider a recursion of which we shall only analyse the essential, i.e. the homogeneous part:

$$
\left[\begin{array}{cc}
\varnothing & -I_{n} \\
A_{i-1}^{2} & B_{i-1}^{2} \\
\varnothing & \varnothing
\end{array}\right]\left[\begin{array}{c}
x_{i-1} \\
x_{i}
\end{array}\right]+\left[\begin{array}{cc}
I_{n} & \varnothing \\
\varnothing & \varnothing \\
A_{i}^{1} & B_{i}^{1}
\end{array}\right]\left[\begin{array}{c}
x_{i} \\
x_{i+1}
\end{array}\right]=0,2 \leq i \leq N-1
$$

(note that (4.7a) is directly found from (4.6) through left multiplication by a suitable nonsingular matrix and setting $b_{i}=0$ ). The form (4.7a) is unbiassed with respect to the increase or decrease of the index sequence

As $\mathrm{BC}$ we have

$$
\left[\begin{array}{cc}
M_{1}^{2} & \varnothing \\
A_{1} & B_{1}^{1} \\
\varnothing & \varnothing
\end{array}\right]\left[\begin{array}{l}
x_{1} \\
x_{2}
\end{array}\right]+\left[\begin{array}{cc}
\varnothing & \varnothing \\
A_{N-1}^{2} & B_{N-1}^{2} \\
\varnothing & M_{N}^{1}
\end{array}\right]\left[\begin{array}{l}
x_{N-1} \\
x_{N}
\end{array}\right]=\left[\begin{array}{c}
b^{2} \\
f_{1}^{1} \\
f_{N-1}^{2} \\
b^{1}
\end{array}\right],
$$

formally written as

$$
\hat{M}_{1}\left[\begin{array}{l}
x_{1} \\
x_{2}
\end{array}\right]+\hat{M}_{N-1}\left[\begin{array}{c}
x_{N-1} \\
x_{N}
\end{array}\right]=\hat{b}
$$

In order to avoid unnecessary complication let us assume that the original recursion (2.1a) involves nonsingular matrices only. Let $\left\{\hat{\Phi}_{i}\right\}_{i=1}^{N-1}$ denote a fundamental solution of (4.7) with $\hat{M}_{1} \hat{\Phi}_{1}+\hat{M}_{N-1} \hat{\Phi}_{N-1}=I$, so $\hat{\Phi}_{i}, \hat{M}_{1}, \hat{M}_{N-1}=\mathbb{R}^{2 n \times 2 n}$.

From (2.3) we see that it is meaningful to define the projections 


$$
P:=M_{1} \Phi_{1}=\left[\begin{array}{ll}
I_{k} & \varnothing \\
\varnothing & \varnothing
\end{array}\right), 1-P=M_{N} \Phi_{N}
$$

Since we here assume that (2.1) is nondefective we can write for the Green's function of (2.1), see (2.4)

$$
\begin{aligned}
& G_{i j}=\Phi_{i} P\left(B_{j} \Phi_{j+1}\right)^{-1}, \quad i \geq j+1 \\
& G_{i j}=\Phi_{i}(I-P)\left(A_{j} \Phi_{j}\right)^{-1} \quad i \leq j
\end{aligned}
$$

We now have

Property 4.10. Let $\mathbf{P}_{1}, \mathbf{P}_{N} \in \mathbb{R}^{n^{2}}$ be such that $\mathbf{P}_{1}=\left[\begin{array}{c}I_{k} \\ \varnothing\end{array}\right], \mathbf{P}_{N}=\left[\begin{array}{c}0 \\ I_{n-k}\end{array}\right]$.

Define $\hat{\Phi}_{i}$ as $\left[\begin{array}{cccc}\hat{\Phi}_{i}^{11} & \hat{\Phi}_{i}^{12} & \hat{\Phi}_{i}^{13} & \hat{\Phi}_{i}^{14} \\ \hat{\Phi}_{i}^{21} & \hat{\Phi}_{i}^{22} & \hat{\Phi}_{i}^{23} & \hat{\Phi}_{i}^{24}\end{array}\right], \hat{\Phi}_{i}^{11}, \hat{\Phi}_{i}^{21}, \hat{\Phi}_{i}^{13}, \hat{\Phi}_{i}^{23} \in \mathbb{R}^{n \times(n-k)}$.

Then for $2 \leq i \leq N-1$ we have

$$
\begin{aligned}
& \hat{\boldsymbol{\Phi}}_{i}^{2 j}=\hat{\boldsymbol{\Phi}}_{i}^{1 j}, j=1, \ldots, 4 \\
& \hat{\boldsymbol{\Phi}}_{i}^{11}=\boldsymbol{\Phi}_{i} \mathbf{P}_{1}, \hat{\boldsymbol{\Phi}}_{i}^{12}=G_{i 1} \mathbf{P}_{N}, \hat{\boldsymbol{\Phi}}_{i}^{13}=G_{i, N-1} \mathbf{P}_{1}, \hat{\boldsymbol{\Phi}}_{i}^{14}=\boldsymbol{\Phi}_{i} \mathbf{P}_{N} .
\end{aligned}
$$

Proof. Write $\hat{\Phi}_{i}=\left[\begin{array}{cc}K_{i}^{11} & K_{i}^{12} \\ K_{i}^{21} & K_{i}^{22}\end{array}\right]$, with square blocks. For $2 \leq i \leq N-2$ we then see $\left[K_{i+1}^{11} K_{i+1}^{12}\right]=\left[K_{i}^{21} K_{i}^{22}\right]$. It follows from the BC that the first $k$ columns of $\left[K_{i}^{11}\right\}$ and the last $(n-k)$ columns of $\left\{K_{i}^{12}\right\}$ form the (normalized) fundamental solution $\left\{\Phi_{i}\right\}$ of (2.1a), (4.1). Furthermore, since the last $(n-k)$ columns of $\left\{K_{i}^{11}\right\}$ satisfy homogeneous $\mathrm{BC}$ and the recursion, except for

$$
\left[A_{1}^{1} K_{1}^{11}+B_{1}^{1} K_{2}^{11}\right]\left[\begin{array}{c}
\varnothing \\
I_{n-k}
\end{array}\right]=I_{n-k},
$$

it is clear that they are identical to the last $n-k$ columns of the Green's function $\left\{G_{i 1}\right\}$. The argument for the first $k$ columns of $\left\{K_{i}^{12}\right\}$ is similar.

Note that for all $j$ the projections like $P_{j}$ in (2.6) are identical to $\hat{P}$, where

$$
\hat{P}=\left[\begin{array}{ll}
I_{n} & \varnothing \\
\varnothing & \varnothing
\end{array}\right] \text {. }
$$

Property 4.12. Let $\left\{\hat{G}_{i j}\right\}$ denote the Green's function of (4.7), Then, using the notation of Property 2.13 , i.e. $\tilde{G}_{i j}=\hat{G}_{i j} \hat{\mathbf{P}}$ (where $\hat{G}_{i j} \in \mathbb{R}^{2 n \times 2 n}$ now) 


$$
\begin{aligned}
& \tilde{G}_{i j}=\left[\begin{array}{cccc}
\Phi_{i} & P & \Phi_{j+1}^{-1} & \varnothing \\
\Phi_{i+1} & P & \Phi_{j+1}^{-1} & \varnothing
\end{array}\right], i \geq j+1
\end{aligned}
$$

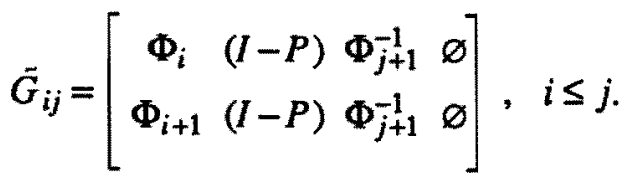

Proof. Apparently, for $i>j$, we have $\bar{G}_{i j}=\hat{\Phi}_{i} \hat{P}\left(\hat{B}_{j} \hat{\Phi}_{j+1}\right)^{+}$(cf. (2.13b)), where

$$
\hat{B}_{j}=\left[\begin{array}{cc}
I_{n} & \varnothing \\
\varnothing & \varnothing \\
A_{j+1}^{1} & B_{j+1}^{1}
\end{array}\right] .
$$

Now $\quad \hat{B}_{j} \hat{\Phi}_{j+1}=\hat{P} \hat{\Phi}_{j+1}=\Phi_{j+1}\left[\begin{array}{c}\left.\mathbf{P}_{1}\left|K \mathbf{P}_{N}\right| L \mathbf{P}_{1} \mid \mathbf{P}_{N}\right\} \\ \varnothing\end{array}\right], \quad$ if $\quad 1 \leq j \leq N-2, \quad$ where $K:=P\left(A_{1} \Phi_{1}\right)^{-1}, L:=(I-P)\left(B_{N-1} \Phi_{N}\right)^{-1}$.

Hence $\hat{B}_{j} \hat{\Phi}_{j+1}$ is of the form $\Phi_{j+1}\left[\begin{array}{cccc}I_{k} & L_{1} & \varnothing & \varnothing \\ \varnothing & \varnothing & L_{2} & I_{n-k} \\ \varnothing & \end{array}\right] I_{n}$, for some $L_{1} \in \mathbb{R}^{k \times(n-k)}$, $L_{2} \in \mathbb{R}^{(n-k) \times k}$.

So

$$
\left(\hat{B}_{j} \hat{\Phi}_{j+1}\right)^{+}=\left[\left(\begin{array}{cc}
\left(I_{k}+L_{1} L_{1}^{T}\right)^{-1} & \varnothing \\
L_{1}^{T}\left(I_{k}+L_{1} L_{1}^{T}\right)^{-1} & \varnothing \\
\varnothing & L_{2}^{T}\left(I_{n-k}+L_{2} L_{2}^{T}\right)^{-1} \\
\varnothing & \left(I_{n-k}+L_{2} L_{2}^{T}\right)^{-1}
\end{array}\right] \Phi_{j+1}^{-1} \mid \varnothing\right]
$$

Substitution in the expression for $\tilde{G}_{i j}$ proves the assertion for $i>j, 2 \leq i \leq N-1$. A similar proof can be carried out for $i \leq j, 2 \leq i \leq N-1$. Further inspection finally shows that for the boundary cases $\tilde{G}_{N j}$ and $\bar{G}_{1 j}$ the assertion is true as well.

It is convenient to associate to the $2 n$-th order system (4.7) quantities as defined in section 2 , but now provided with a cap to avoid confusion. Hence the Green's function $\hat{G}_{i j}$ (see Property 4.12) actually consists of two parts (cf. (2.7)ff), viz. $\hat{G}_{i j} \hat{P}_{1}=\tilde{G}_{i j}$ and $\hat{G}_{i j}\left(I-\hat{P}_{1}\right)=: \hat{F}_{i j}$. To the latter "parasitic part" we can associate projection matrices $\hat{Q}_{1}$ and $I-\hat{Q}_{1}$, defined for the larger system just like in Property 2.15(iii), realizing that they are index independent. The proof of the following Property about "parasitic solutions" is immediate.

\section{Property 4.13.}


(i) The projection matrix $\hat{Q}_{j}$ is given by $\left[\begin{array}{lll}\varnothing & \varnothing & \varnothing \\ \varnothing & I_{k} & \varnothing \\ \varnothing & \varnothing & \varnothing\end{array}\right]\lfloor n$.

(ii) $F_{i}^{i}=\left[\begin{array}{cc}\varnothing & G_{i j} \\ \varnothing & G_{i+1, j}\end{array}\right]$.

Corollary 4.14. The Green's function for $(4.7 \mathrm{a}, \mathrm{b})$ is given by

$$
\begin{aligned}
& {\left[\begin{array}{cc}
\Phi_{i} P \Phi_{j+1}^{-1} & G_{i j} \\
\Phi_{i+1} P \Phi_{j+1}^{-1} & G_{i+1 j}
\end{array}\right], i \geq j+1} \\
& {\left[\begin{array}{cc}
-\Phi_{i}(I-P) \Phi_{j+1}^{-1} & G_{i j} \\
-\Phi_{i}(I-P) \Phi_{j+1}^{-1} & G_{i+1 j}
\end{array}\right], i \leq j .}
\end{aligned}
$$

Of course, the results of this section might also be obtained by manipulating directly on the matrix resulting from $(4.7 \mathrm{a}, \mathrm{b})$ in relation to $(4.2)$. However, the construction in this section demonstrates the validity of our analysis given in Section 3 .

From Theorem 3.11 we can now see that the (inflated) one-step system $(4.7 \mathrm{a}, \mathrm{b})$ has a dichotomic fundamental solution. 


\section{Decoupling and singular matrices}

In this section we consider the BVP (2.1), (2.2), assuming that it is well-conditioned, but allowing any of the matrices involved to be singular.

A general decoupling method which employs orthogonal matrices was given in [8]. We first describe it here briefly: Let $Q_{0}$ be an orthogonal matrix. Then perform a QU-decomposition

$$
A_{1} Q_{1}=R_{1} U_{1}
$$

(i.e. $R_{1}$ orthogonal and $U_{1}$ (block) upper triangular). Next, perform a "(block) UQdecomposition" on $R_{1}^{-1} B_{1}$, i.e.

$$
R_{1}^{-1} B_{1}=V_{1} Q_{2}^{-1}
$$

where $V_{1}$ is (block) upper triangular. Initially one can take both $U_{1}$ and $V_{1}$ to be upper triangular. The block form follows from the construction in Theorem 5.6. We can continue this process giving at the $i$-th step

$$
\begin{aligned}
& A_{i} Q_{i}=: R_{i} U_{i} \\
& R_{i}^{-1} B_{i}=: V_{i} Q_{i+1}^{-1}
\end{aligned}
$$

By defining

$$
\begin{aligned}
& \bar{M}_{1}:=M_{1} Q_{1} \\
& \bar{M}_{N}:=M_{N} Q_{N} \\
& \bar{x}_{i}:=Q_{i}^{-1} x_{i} \\
& \bar{f}_{i}:=R_{i}^{-1} f_{i},
\end{aligned}
$$

we obtain an upper triangular recursion

$$
U_{i} \bar{x}_{i}+V_{i} \bar{x}_{i+1}=\bar{f}_{i}
$$

In order to employ the decoupling for separate computation of non-increasing and non-decreasing modes, we need appropriate blocks of $U_{i}$ and $V_{i}$ to be nonsingular. In the following theorem we shall show that a suitable permutation gives block upper triangular $U_{i}$ and $V_{i}$, which allow for a globally meaningful decoupling.

Theorem 5.6. Let $\operatorname{rank}\left(A_{i}\right)=k_{i}$ and $\operatorname{rank}\left(B_{i}\right)=n-l_{i}$. Then there exists an orthogonal matrix $Q_{1}$ such that $U_{i}$ has zero lower right $\left(n-k_{i}\right) \times\left(n-k_{i}\right)$ blocks and $V_{i}$ has zero upper left $l_{i} \times l_{i}$ blocks. Moreover $l_{i} \leq k_{i}$ for all $i$ and there exists a $k$, with $l_{i} \leq k \leq k_{i}$ for all such that the upper left $k \times k$ blocks of the $U_{i}$ and the lower $(n-k) \times(n-k)$ blocks of the $V_{i}$ are nonsingular.

Proof. We shall use induction. We can always choose the columns in $Q_{i}$ such that the first $k_{1}$ 
columns are orthogonal to $\operatorname{Null}\left(A_{1}\right)$, so the last $\left(n-k_{1}\right)$ rows of $U_{1}$ are zero. Since the BVP is well-posed, i.e. the "multiple shooting" matrix is nonsingular it follows that the last $\left(n-k_{1}\right)$ rows of $R_{1}^{-1} B_{1}^{-1}$ must have full rank, so the lower right $\left(n-k_{1}\right) \times\left(n-k_{1}\right)$ block of $V_{1}$ is nonsingular. By a suitable choice of $R$, we can guarantee that $V_{1}$ has zeros on the first $l_{1}$ diagonal positions, where $l_{1}$ is such that $\operatorname{rank}\left(B_{1}\right)=n-l_{1}$.

Now let the statement be true for $i-1$. Due to the zero $l_{i-1} \times l_{i-1}$ left upper block of $V_{i-1}$, the corresponding block of $U_{i}$ must be nonsingular, i.e. $k_{i} \geq l_{i-1}$. It is always possible to permute the last $n-l_{i-1}$ columns of $B_{i-1}$ and $A_{i}$ such that $U_{i}$, when singular, i.e. of $k_{i} \leq n$, is such that its right lower $\left(n-k_{i}\right) \times\left(n-k_{i}\right)$ is zero; this affects only the orthogonal matrix $Q_{i}$ : if the permutation $P_{i}$, say, then one should replace $Q_{i}$ by $P_{i} Q_{i}$.

The zero block in $U_{i}$ implies a nonsingular block at the same position in $V_{i}$ hence $l_{i} \leq k_{i}$. By permuting rows of $A_{i}$ and $B_{i}$ it can be assured that the left upper $\left(l_{i} \times l_{i}\right)$ block of $V_{i}$ is zero. The existence of the integer $k$ follows from the construction above.

The proof of the preceding theorem actually gives a constructive method for finding a partioning integer $k$ and $\left\{Q_{i}, R_{i}\right\}$. We can go a step further and construct $Q_{i}$ such that it produces an appropriately ordered diagonal of the $U_{i}$ and $V_{i}$ as far as the nondisappearing parts of the fundamental solution of $(5.5),\left\{\bar{\Phi}_{i}\right\}_{i=1}^{N}$ say, are concemed.

Now let $\bar{k}$ be the largest possible integer such that for all $i$, the $\bar{k} \times \bar{k}$ left upper blocks of $U_{i}$ are nonsingular (see Theorem 5.6).

Let us denote by $\bar{U}_{i}$ and $\tilde{V}_{i}$ the $\bar{k} \times \bar{k}$ left upper blocks of $U_{i}$ and $V_{i}$ respectively. This induces the recursion

$$
\tilde{U}_{i} \tilde{x}_{i}+\tilde{V}_{i} \tilde{x}_{i+1}=0
$$

Property 5.8. Let $\bar{l}:=\max l_{i}$. Then there exists a matrix solution $\left\{\tilde{\Psi}_{i}\right\}$ of $(5.7)$, so $\tilde{\Psi}_{i} \in \mathbb{R}^{\bar{k} \times \bar{k}}$ for all $i$ and a projection $\bar{P}$, with $\operatorname{rank}(\bar{P}) \leq \bar{k}-\bar{l}$ such that

$$
\begin{aligned}
& \left\|\tilde{\Psi}_{i} \bar{P} \tilde{\Psi}_{j}^{+}\right\| \leq\left(\kappa+4 \kappa^{2}\right) \gamma_{j}, \quad i \leq j \\
& \left\|\tilde{\Psi}_{i}(I-\bar{P}) \tilde{\Psi}_{j+1}^{+}\right\| \leq\left(\kappa+4 \kappa^{2}\right) \gamma_{j}, \quad i \geq j+1, \\
& \text { where } \gamma_{j}=\max \left(\left\|A_{j}\right\|,\left\|B_{j}\right\|\right) \text {. }
\end{aligned}
$$

Proof. We use the orthogonal invariance of the 2-norm to see that the problem (5.3), (5.5) induces a matrix with the same bound for its inverse as (2.1); hence because of the orthogonality of the transformation $\left\{Q_{i}\right\}$ and $\left\{R_{i}\right\}$ the estimates essentially follow from Theorem 3.11 , constructing $\tilde{P}$ like $P$ in (3.5).

From Property 5.8 we deduce that we can choose the matrix $Q_{1}$ such that the nondecreasing modes, cf. $\left\{\bar{\Psi}_{i} \tilde{P}\right\}$ in Property 5.8, appear before the nonincreasing ones in $\left[\Psi_{i}\right\}$, i.e. $\bar{P}$ is of the 
form diag $(0,0, \ldots, 0,1, \ldots, 1)$. This then finally gives us the result that there exists a stable decoupling, also in the rank deficient case. 


\section{Examples}

In this section we give two-examples to demonstrate the preceding analysis

\section{Example 6.1. Consider the ODE}

$$
\frac{d x}{d t}=\left[\begin{array}{cc}
c^{2} \lambda+s^{2} \mu & c s(\mu-\lambda) \\
c s(\mu-\lambda) & s^{2} \lambda+c^{2} \mu
\end{array}\right] x+f(t),
$$

where $c=\cos t, s=\sin t$ and $f$ is chosen such that $x(t)=(\cos t, \sin t)$ is a solution. As BC we take

$$
\left[\begin{array}{ll}
1 & 0 \\
0 & 0
\end{array}\right] x(0)+\left[\begin{array}{ll}
0 & 0 \\
1 & 0
\end{array}\right] x(\pi)=\left[\begin{array}{c}
1 \\
-1
\end{array}\right]
$$

For $\lambda$ large positive and $\mu$ large negative this constitutes a stiff BVP, having layers at both ends of the interval $[0, \pi]$. Therefore we use the midpoint schema on a nonuniform interval: Writing (6.1) as

$$
\frac{d x}{d t}=A(t) x+f
$$

we have at the grid point $t_{i}$

$$
\left[I-\frac{1}{2} h A_{i+\frac{1}{2}}\right] x_{i+1}=\left[I+\frac{1}{2} h A_{i+\frac{1}{2}}\right] x_{i}+h f_{i+\frac{1}{2}},
$$

where $A_{i+\frac{1}{2}}:=A\left(\frac{1}{2}\left(t_{i+1}+t_{i}\right)\right)$.

As grid points we use $\frac{j}{10}, j=0, \ldots, 30$ and moreover in the left layer: $t=\frac{1}{1200}, \frac{1}{600}, \frac{1}{400}, \frac{1}{300}, \frac{1}{250}, \frac{1}{200}, \frac{1}{150}, \frac{1}{100}, \frac{1}{50}, \frac{1}{25}$ and in the right layer the points $t=\pi-\frac{j}{10}$, for $j=0 ; \cdots, 30$.

In Table 6.1 we display some typical values for $U_{i}$ and $V_{i}$ 


\begin{tabular}{lllll}
\multicolumn{1}{c}{$t$} & \multicolumn{2}{c}{$U_{i}$} & \multicolumn{2}{c}{$V_{i}$} \\
0.04 & 1.4999 & -0.0125 & 0.5000 & $.610^{-18}$ \\
& 0 & $.710^{-10}$ & 0 & 2.0000 \\
0.10 & 1.9998 & 0.0225 & $.910^{-16}$ & -0.0225 \\
& 0 & 1.0001 & 0 & 2.9999 \\
& & & & \\
1.6 & 6.1809 & -1.5710 & 4.1933 & -3.0522 \\
& 0 & 8.7366 & 0 & 10.4927 \\
& & & & \\
2.9 & 6.1809 & -1.5710 & 4.1933 & -3.0522 \\
& 0 & 8.7366 & 0 & 10.4927 \\
& & & & $.910^{-15}$ \\
3.1316 & 1.9960 & 0.1097 & -0.1100 \\
& 0 & 1.0020 & 0 & 2.9980 \\
& & & & \\
3.1333 & 1.4998 & 0.0225 & 0.5000 & $.410^{-15}$ \\
& 0 & $.2310^{-13}$ & 0 & 2.0000
\end{tabular}

Table 6.1.

For $t=0.04,3.1333$ we see that $U_{i}$ is almost singular and similarly $V_{i}$ for $t=0.10,3.1316$; latter points mark the end of the layers, and if the decoupling is done adequately this should not have a noticeable global effect (cf. [1]). Though, clearly, $A$ is not constant, the matrices $U_{i}$ and $V_{i}$ appear to be almost constant (due to the fact that $h \lambda,|h \mu|$ are "large", a well-known by-product of distable methods, see [3]). It is worth noting that the dichotomy can be deduced from the quotient of diagonal elements of $U_{i}$ and $V_{i}:\left|\left(U_{i}\right)_{22} /\left(V_{i}\right)_{22}\right|<1$ and $\left|\left(V_{i}\right)_{11} /\left(U_{i}\right)_{11}\right|<1$.

In tabel 6.2 we have displayed the discrete fundamental solution $\bar{\Phi}_{i}$ of the transformed recursion 5.5 for some values of $t_{i}$, and in the last column the absolute error vector (after transforming back). 


\begin{tabular}{|c|c|c|c|}
\hline$t$ & \multicolumn{2}{|c|}{$\bar{\Phi}_{i}$} & error \\
\hline \multirow[t]{2}{*}{0} & $0.36510^{-39}$ & $0.344 \quad 10^{-2}$ & 0 \\
\hline & 0 & 1.00 & $0.27410^{-2}$ \\
\hline \multirow[t]{2}{*}{0.04} & $0.29910^{-38}$ & $-0.74210^{-19}$ & $0.63910^{-4}$ \\
\hline & 0 & $.49510^{-17}$ & $0.18110^{-5}$ \\
\hline \multirow[t]{2}{*}{0.10} & $0.63910^{-22}$ & $-0.18810^{-18}$ & $0.12110^{-2}$ \\
\hline & 0 & $0.16510^{-17}$ & $0.19510^{-4}$ \\
\hline \multirow[t]{2}{*}{1.6} & $0.44710^{-19}$ & $-0.26310^{-19}$ & $0.26510^{-4}$ \\
\hline & 0 & $0.72810^{-19}$ & $0.12510^{-2}$ \\
\hline \multirow[t]{2}{*}{2.9} & $0.10210^{-16}$ & $-0.11810^{-20}$ & $0.19510^{-2}$ \\
\hline & 0 & $0.56010^{-20}$ & $0.20510^{-4}$ \\
\hline \multirow[t]{2}{*}{3.1316} & 0.122 & $-0.12610^{-22}$ & $0.22210^{-6}$ \\
\hline & 0 & $0.83710^{-21}$ & $0.351 \quad 10^{-6}$ \\
\hline \multirow[t]{2}{*}{3.1333} & 0.367 & $0.77610^{-37}$ & $0.35110^{-6}$ \\
\hline & 0 & $0.96510^{-35}$ & $0.19810^{-6}$ \\
\hline \multirow[t]{2}{*}{3.1416} & 1.000 & 0 & 0 \\
\hline & 0 & $0.12810^{-35}$ & $0.48110^{-7}$ \\
\hline
\end{tabular}

Table 6.2.

The table shows that there is a nice layer resolution indeed; moreover the global error is of a proper order. These computations are essentially based on employing the stable decoupling.

Example 6.2. Consider the second order DAE

(6.4) $E \dot{x}=A x+q$,

where $E$ is the (constant, singular) matrix $\left[\begin{array}{ll}1 & 0 \\ 0 & 0\end{array}\right]$.

Introducing an additional variable $y$, we can write

$$
\left\{\begin{array}{l}
E \dot{x}=y \\
E y=A x+q
\end{array}\right.
$$

For this simple index 1 probleem, we can use the trapezoidal method with grid spacing $h$ resulting in (cf. [5]) 


$$
A_{i} z_{i}+B_{i} z_{i+1}=b_{i}, \quad i=0, \ldots, N-1
$$

where

$$
A_{i}:=\left[\begin{array}{cc}
-\frac{1}{h} E-\frac{1}{2} E \\
\varnothing & \varnothing
\end{array}\right], B_{i}:=\left[\begin{array}{cc}
-\frac{1}{h} E-\frac{1}{2} E-\eta \\
-A & E
\end{array}\right],
$$

and $z_{i}=\left[\begin{array}{l}x_{i} \\ y_{i}\end{array}\right]$, with $x_{i} \doteq x(i h), y_{i} \doteq y(i h)$.

Assume we have a $B C$ for (6.4)

$$
M_{a} x(a)+M_{b} x(b)=c,
$$

where $M_{a}, M_{b} \in \mathbb{R}^{1 \times 2}$.

Then a complete set of $B C$ for (6.6) is given by (cf. [5])

$$
M_{0} z_{0}+M_{N} z_{n}=d
$$

where

$$
M_{0}:=\left[\begin{array}{cc}
M_{a} & I-E \\
-A & E
\end{array}\right], M_{N}:=\left[\begin{array}{cc}
M_{b} & \varnothing \\
\varnothing & \varnothing
\end{array}\right], d:=\left[\begin{array}{c}
c \\
0 \\
q(a)
\end{array}\right]
$$

The BVP (6.6), (6.9) has a unique solution. A typical block row in the matrix resulting from the set of equations for $\left\{z_{i}\right\}$ is given by

$$
\left[\begin{array}{cccc|cccc}
-\frac{1}{h} & 0 & -\frac{1}{2} & 0 & -\frac{1}{h} & 0 & \frac{1}{2} & 0 \\
0 & 0 & 0 & 0 & 0 & 0 & 0 & -1 \\
0 & 0 & 0 & 0 & * & * & 1 & 0 \\
0 & 0 & 0 & 0 & * & * & 0 & 0
\end{array} \mid \varnothing\right]
$$

(where * denotes an element of $A$ ). In view of the algorithm outlined in section 5 , we note that whatever choice of $Q_{i}$ is made, $R_{i} U_{i}$, so $U_{i}$, will have zeros in the last three rows. Hence we can take $R_{i}=I$. As for the matrix $B_{i}$ we note that it is nonsingular if $A$ is (unless $h$ is such that $\left.a_{11}+2 / h=a_{12} a_{21} / a_{22}\right)$. Hence we can take $Q_{i+1}$ simply such that $B_{i} Q_{i+1}$ is upper triangular (by Householder's method e.g.). The resulting matrices $U_{i}$ and $V_{i}$ induce a simple decoupling: The dimension $k$ of the nonsingular diagonal block is 1 . From this it follows that the projection matrix $\bar{P}$ as in Property (5.8) must equal

$$
\bar{P}=\left[\begin{array}{ll}
1 & \varnothing \\
\varnothing & \varnothing
\end{array}\right]
$$

Hence it follows that the parasitic solutions generated by the Green's function are zero, except at the point where they arise. This then implies numerical stability of the decoupling if only the decoupled scalar recursion employing the first diagonal elements of $U_{i}$ and $V_{i}$ is used in a stable 
direction. The existence of a stable direction follows directly from the well conditioning of the DAE (6.4), (6.8) (cf. [4]). 


\section{References}

[1] U.M. Ascher, R.M.M. Mattheij, General Framework, Stability and Error Analysis for Numerical Stiff Boundary Value Methods, Num. Math 54 (1988), 355-372.

[2] U.M. Ascher, R.M.M. Mattheij, R.D. Russell, Numerical Solution of Boundary Value Problems for Ordinary Differential Equations, Prentice-Hall, 1988, Englewood-Cliffs.

[3] R. England, R.M.M. Mattheij, Boundary Value Problems and Dichotomic Stability, SIAM J. Numer. Anal. 25 (1988), 1037-1054.

[4] Griepentrog, R. März, Differential Algebraic Equations and their Numerical Treatment, Tubner, Reipzig (1986).

[5] G.H. Golub, C.F. van Loan, Matrix Computations, John Hopkins Univ. Press, Baltimorre, 1983.

[6] F.R. de Hoog, R.M.M. Mattheij, On dichotomy and well-conditioning in BVP, SIAM J. Numer. Anal. 24 (1987), 89-105.

[7] R.M.M. Mattheij, Estimates for the errors in the solutions of linear boundary value problems, due to perturbations, Computing 27 (1981), 299-318.

[8] R.M.M. Mattheij, Decoupling and stability of algorithms for boundary value problems, SIAM Review 27 (1984), 314-331. 